\title{
Implementation of Fuzzy Logic Control System on Rotary Car Parking System Prototype
}

\author{
Nanang Ismail ${ }^{1}$, Iim Nursalim², Hendri Maja Saputra ${ }^{3}$, Teddy Surya Gunawan ${ }^{4}$ \\ ${ }^{1,2}$ Electrical Engineering Department, Faculty of Science and Technology, UIN Sunan Gunung Djati \\ Jalan A.H Nasution 105, Bandung, Indonesia \\ ${ }^{3}$ Research Center for Electrical Power and Mechatronics, Indonesian Institute of Sciences \\ Jl. Cisitu No.21/154D Sangkuriang - Bandung 40135, Indonesia \\ ${ }^{4}$ Electrical and Computer Engineering Department, Kulliyyah of Engineering International Islamic University Malaysia, \\ Jalan Gombak, 53100 Kuala Lumpur, Malaysia
}

\begin{abstract}
Article Info
Article history:

Received Apr 9, 2018

Revised May 20, 2018

Accepted Jul 11, 2018

\section{Keywords:}

Control

Fuzzy logic

Kinematic

Rotary Car Parking System

ABSTRACT

Rotary car parking system (RCPS) is one of the effective parking models used in the metropolitan area because the mechanical parking system is designed vertically to conserve the land usage. This paper discussed the implementation of fuzzy logic with the Sugeno Inference Model on the RCPS miniature control system. The research started with kinematics analysis and a mathematical model was derived to determine the slot position and optimal power requirements for each condition. Furthermore, the Fuzzy Inference model used was the Sugeno Model, taking into account two variables: distance and angle. These two variables were selected because in the designed miniature RCPS there will be rotational changes of rotation and rotation in turn. Variable distance was divided into four clusters, such as Zero, Near, Medium and Far. While the angle variables were divided into four clusters as well, such as Zero, Small, Medium, and Big. The test results on a miniature RCPS consisting of six parking slots showed that fuzzy based control provided better results when compared to conventional systems. Step response on the control system without fuzzy control showed the rise time value of 0.58 seconds, peak time of 0.85 seconds, settling time of 0.89 , percentage overshoot of $0.20 \%$, and steady state error of $4.14 \%$. While the fuzzy control system provided the rise time value of 0.54 seconds, settling time of 0.83 seconds, steady state error of $2.32 \%$, with no overshoot.
\end{abstract}

Copyright (c) 2018 Institute of Advanced Engineering and Science. All rights reserved.

Corresponding Author:

Nanang Ismail,

Electrical Engineering Department, Faculty of Science and Technology,

UIN Sunan Gunung Djati,

Jalan A.H Nasution 105, Bandung, Indonesia.

Email: nanang.is@uinsgd.ac.id

\section{INTRODUCTION}

One of the solution to the problem of limiting parking space is the mechanical parking system. On the other hand, mechanical parking is a new type of parking system which uses mechanical and automated system with an electric power source to minimize parking space used while maximizing number of vehicles that can be parked safely. The mechanical-based parking system has several types, including rotary parking system, multilevel car parking, optima car parking, and speedy tower car parking [1]. Each parking model, such as integrated car parking solution, automated car parking, multi-level parking and rotary parking system, has different advantages and disadvantages. The parking system with rotation model can accommodate 6-15 cars with minimal land scales [2]. It uses a gear and chain grille on the motor to drive the car storage slot. 
Generally, modern mechanical systems require an optimal control system to control the system, including fuzzy logic control [3]. Currently, the fuzzy control system has also been applied in various areas of life such as for water control [4], automatic rail operation [5], automatic crane container operation [6], elevator control [7], nuclear reactor control [8], power control [9], and autonomous parallel parking [10, 11]. In the process of utilizing fuzzy logic, there are some things that must be considered, one of which is how to process input into output through fuzzy inference system. The fuzzy inference method is a way of formulating the input mapping given to an output. This process involves membership functions, logic operations, and IF-THEN rules [12]. The result of this process will produce a system called Fuzzy Inference System (FIS). In fuzzy logic, there are available several types of FIS included Mamdani [12-14], Sugeno [15-17], and Tsukamoto [19].

The fuzzy control system aims to eliminate overshoot and minimize errors [18, 19]. The RCPS control system requires controls that minimize overshoot and error, due to the demands of maximum accuracy. This paper discusses the implementation of fuzzy control system with Sugeno Model for controlling Rotary Car Parking system (RCPS). The system is tested on a miniature RCPS consisting of 6 space slots for vehicles. The fuzzy control system is applied after a kinematic analysis of the designed RCPS [20]. The response of the RCPS miniature control system with Fuzzy will then be compared with the conventional control system .

\section{PROPOSED MODEL FOR RCPS WITH KINEMATIC ANALYSIS}

Rotary car parking system requires positioning and supply calculation of the right power to avoid failure. Determining the location and distance of the slot will affect the motor speed and power requirements that must be provided. Power and speed requirements are also affected by the number, size, and load of each slot. Kinematic analysis is required by creating a mathematical model to determine the slot position and optimal power requirement in each condition because in RCPS there is a change of rotation motion into translational motion. The model used in performing kinematic analysis to create a RCPS mathematical model is the rotary car parking scheme shown in Figure 1 [20].

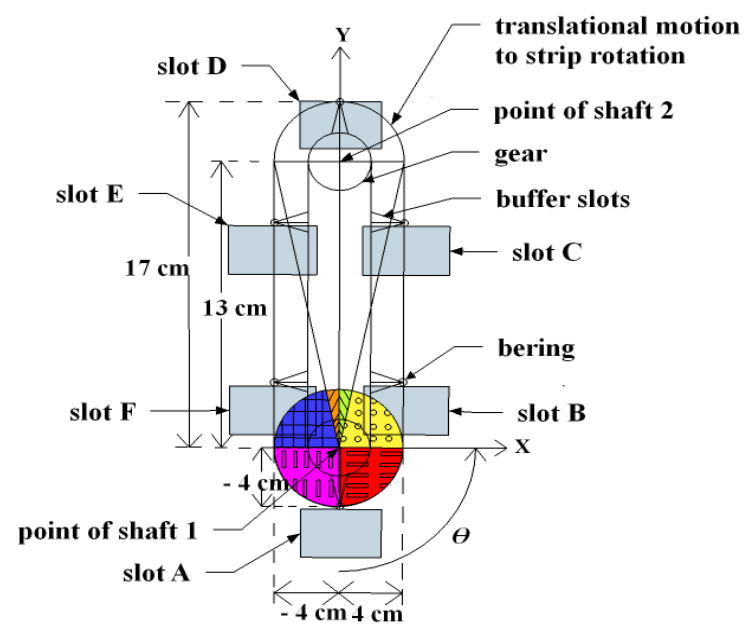

Figure 1. Scheme of rotary car parking system

In Figure 1, it is assumed that one slot can only be occupied by one car. In the parking system mechanism as shown in Figure 1, there are angles $\theta$ and slot positions in coordinates $(x, y)$. The RCPS divides its angle into six parts, i.e. $\left(0^{\circ}-90^{\circ}\right),\left(90^{\circ}-166^{\circ}\right),\left(166^{\circ}-180^{\circ}\right),\left(180^{\circ}-194^{\circ}\right),\left(194^{\circ}-270^{\circ}\right)$, and $\left(270^{\circ}-360^{\circ}\right)$. The division is based on the change of the rotational motion to the translational motion, vice versa [20].

The equation for determining the coordinate position of the $x$ and $y$ axes is obtained by trigonometric equations. The equation is also proved by manual measurement on the sketch by using ruler and arc so as to produce Equation (1) and (2) [20]. 


$$
\begin{aligned}
& x= \begin{cases}x_{1}=x_{\text {max }} \cdot \sin \theta & \left(0 \leq \theta \leq 90^{\circ}\right) \&\left(270^{\circ} \leq \theta<360^{\circ}\right) \\
x_{2}=\left(x_{\max } \cdot \sin \theta\right)+x_{\text {max }} \cdot(1-\sin \theta) & \left(90^{\circ}<\theta<166^{\circ}\right)\left(194^{\circ}<\theta<270^{\circ}\right) \\
x_{3}=y_{\text {max }} \cdot \sin \theta & \left(166^{\circ} \leq \theta \leq 194^{\circ}\right)\end{cases} \\
& y= \begin{cases}y_{1}=y_{\text {max }} \cdot \cos \theta & \left(0 \leq \theta \leq 90^{\circ}\right) \&\left(270^{\circ} \leq \theta<360^{\circ}\right) \\
y_{2}=-y_{\text {max }} \cdot(\cos \theta)^{5} & \left(166^{\circ} \leq \theta<194^{\circ}\right) \\
y_{3}=y_{\text {max }} \cdot \sin \theta & \left(90^{\circ}<\theta<166^{\circ}\right)\left(194^{\circ}<\theta<270^{\circ}\right) .\end{cases}
\end{aligned}
$$

A mathematical model was also derived to calculate the power requirements to hold still the RCPS position. If the resources provided are not in accordance with the needs of the system, then the system will not work or will be damaged. Therefore, the power source adjustment is very important so that the system can work well. This equation has been discussed in paper [20], where the mathematical model for calculating minimum power requirements for holding RCPS is represented by Equation (3).

$$
P \geq m_{s} . g . \omega .2 \pi \frac{\left(m_{c A} \cdot r_{A}+m_{C B} \cdot r_{B}+m_{c C} \cdot r_{C}+m_{c D} \cdot r_{D}+m_{c E} \cdot r_{E}+m_{c F} \cdot r_{F}\right)}{\eta \cdot 60},
$$

where $\omega$ is the rotational speed of the motor (rpm), $m_{s}$ is the slot mass, $m_{c i}$ is the car mass in the slot $i$, and $r_{i}$ is $x$ value of the gravity drop point for each position of the slot joint $i$ with $i=[A, B, C, D, E]$. Slot B and $\mathrm{C}$ have $r=x_{\max }$, i.e. $4 \mathrm{~cm}$, slot $\mathrm{E}$ and $\mathrm{F}$ is $-4 \mathrm{~cm}$, and slot $\mathrm{D}$ is $0 \mathrm{~cm} \mathrm{[20].}$

The kinematic equation is an equation used to find the slot position in $x$ and $y$ coordinates by inserting the angle value. In addition, the kinematics equation can also predict the minimum power required by the motor to withstand the mechanical load of RCPS. The kinematic modelling has been evaluated and discussed in [20]. The simulation parameters is shown in Table 1.

Table 1. Simulation Parameters [3]

\begin{tabular}{clc}
\hline No & \multicolumn{1}{c}{ Parameter } & Value \\
\hline 1 & Mass of cars $\left(m_{c}\right)$ & $1800 \mathrm{~kg}$ \\
2 & Mass of slot $\left(m_{s}\right)$ & $300 \mathrm{~kg}$ \\
3 & Rotation speed $(\omega)$ & $0.1 \mathrm{~m} / \mathrm{s}$ \\
4 & Motor Efficiency $(\eta)$ & $90 \%$ \\
\hline
\end{tabular}

Based on Table 1, we conducted extensive simulation. Equation (1) and (2) were used to obtained the position of slot joint coordinates $(x, y)$. The coordinate positions are obtained from the angle ranging from $0^{\circ}$ to $360^{\circ}$. The calculated values are then plotted and compared to the manual trajectory as shown in Figure 2.

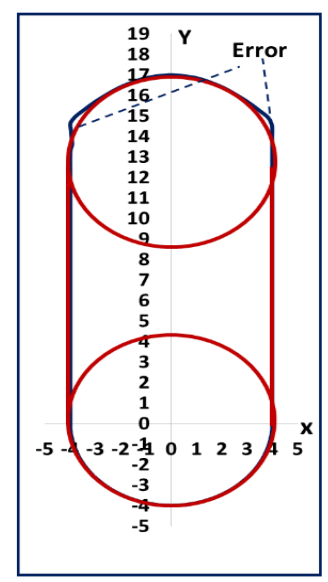

Figure 2. Simulation error

Figure 2 shows that Equation (1) and (2) are valid.The red line is the manual trajectory, while the black line is obtained from Equation (1) and (2). There are two small error visible, which is the error in the 
positive $\mathrm{x}$-axis in the range of $163^{\circ}$ to $175^{\circ}$, and the error in the negative $\mathrm{x}$-axis in the range of $185^{\circ}$ to $197^{\circ}$. Based on the power calculation as in Equation (3) with simulation parameters as shown in Table 1, there are four position of highest power values, i.e. 492.73 watt. The highest power required is obtained when each parking slot is occupied by car with weight of $1800 \mathrm{~kg}$. The unbalanced mechanical position causes the power requirement to be higher, while the balanced position requires power of 0 watt [20].

\section{PROPOSED RCPS MINIATURE}

Miniature RCPS is implemented based on a predefined scheme with a size proportional to the sketch. RCPS uses a pair of gears, with a 24 Volt DC motor as a driver. Installation of connecting iron between the left gear and right gear is done using electric welding, so that the gear shaft point is stronger if the load is excessive because the gear is the pivot point. The connector between the lower gear and the top gear uses the chain bars. The aluminum plate is used as a platform for vehicle storage slots. The actual designed miniature RCPS can be seen in Figure 3.

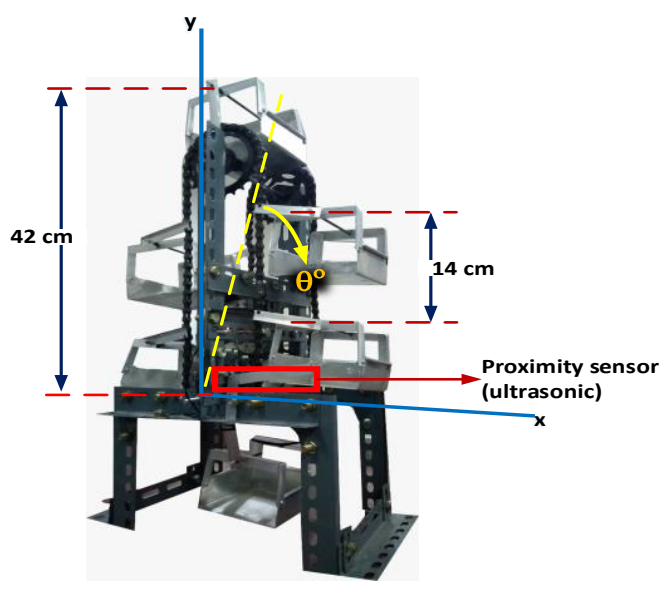

Figure 3. Actual Implementation of Miniature RCPS

Ultrasonic sensor with type HC SR04 is used to read the distance of the object in the form of slot from the reference point. The reference point is the $\mathrm{x}$ axis, parallel to the slot position $\mathrm{B}$ in the schematic of Figure 1. This sensor will detect the approximate distance of the object (slot) to the $\mathrm{X}$-axis. The ultarsonic sensor will emit an ultrasonic signal to the object and reflect back to the sensor. The distance reading mechanism with this sensor can be illustrated in Figure 4.

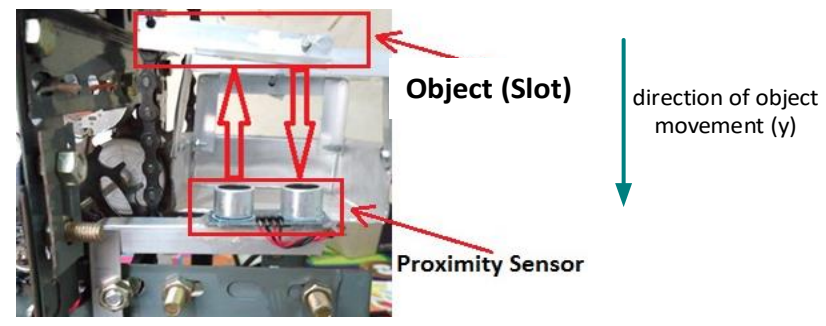

Figure 4. Distance reading mechanism by using ultrasonic sensors

Meanwhile, to read the turning angle, an absolute rotary encoder sensor is used. The principle of this sensor works by reading the hole on the sensor disk which is then converted in the form of data. The absolute rotary encoder in this study was coupled to the mechanical side of the gear chain in order to observe the rotational position of the gear as shown in Figure 5. The resulting data of rotary encoder is mechanical rotary position in the form of decimal number. Data in the form of decimal numbers can be converted into angular form. 


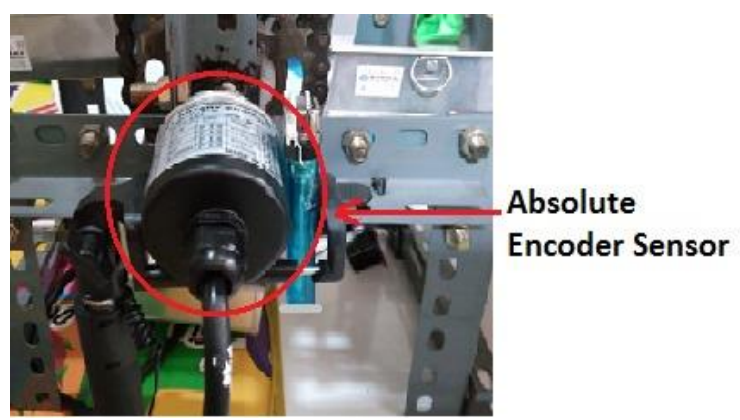

Figure 5. Absolute encoder sensor

\section{DESIGN OF CONTROL SYSTEM}

The control system in this research is fuzzy logic based. Fuzzy logic itself is widely used as intelligent control because it can determine the desired variable by a particular approach. This control system will adjust the system's rotational speed due to distance change. With this fuzzy control, the speed of the motor will be adjusted to the distance, when to turn fast, medium, slow, and stop. The control system was developed using Atmega 328P microcontroller.

\subsection{Realization of Fuzzy Inference System (FIS)}

FIS in this study using Takagi Sugeno Kang (Sugeno) reasoning method. The process of this fuzzy inference system consists of crisp input, fuzzification, evaluation rule, defuzzification, and crisp output. There are two input variables of this system, those are distances $(y)$ and angle $(\theta)$. The distance variable shows the distance between the observed object against the sensor on the $x$-axis. The limitation of each parameter in the membership function is based on the mechanical scale used. The distance is measured by the ultrasonic sensor previously shown at Figure 4. The object, in the form of a slot, moves down near the sensor from farthest distance of $14 \mathrm{~cm}$ to $0 \mathrm{~cm}$. While the angular variable is the magnitude of the angle left by the slot movement with zero point reference (position of the encoder sensor). Membership funtion distance is arranged in line and triangle functions. Object distance with sensor has 4 membership functions as shown in Figure 6. The membership function criteria for distance are shown in Equation (4) to (7).

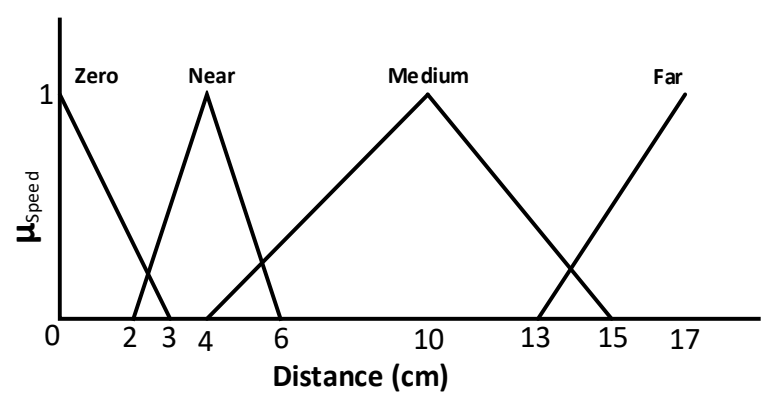

Figure 6. Distance Membership Function

a. Zero

$$
\mu_{\text {zero }}(y)=\left\{\begin{array}{lr}
\frac{3-y}{3-0} ; & 0 \leq y \leq 3 \\
0 ; & y \geq 3
\end{array}\right.
$$

b. Near

$$
\mu_{\text {near }}(y)=\left\{\begin{array}{lc}
0 ; y \leq 2 \text { and } y \geq 6 \\
\frac{y-2}{4-2} ; \quad 2 \leq y \leq 4 \\
\frac{6-y}{6-4} ; \quad 4 \leq y \leq 6
\end{array}\right.
$$


c. Medium

$$
\mu_{\text {medium }}(y)=\left\{\begin{array}{l}
0 ; y \leq 4 \text { and } y \geq 15 \\
\frac{y-4}{10-4} ; 4 \leq y \leq 10 \\
\frac{15-y}{15-10} ; 10 \leq y \leq 15
\end{array}\right.
$$

d. Far

$$
\mu_{\text {far }}(x)=\left\{\begin{array}{c}
\frac{y-17}{17-13} ; \quad 3 \leq y \leq 17 \\
0 ; \quad y \leq 13 \text { and } y \geq 17
\end{array}\right.
$$

Meanwhile, the angular funtion membership is composed by triangle and trapezoidalfunctions. The angle has four membership functions as shown in Figure 7. The membership function criteria for angles are shown in Equation (8) to (11).

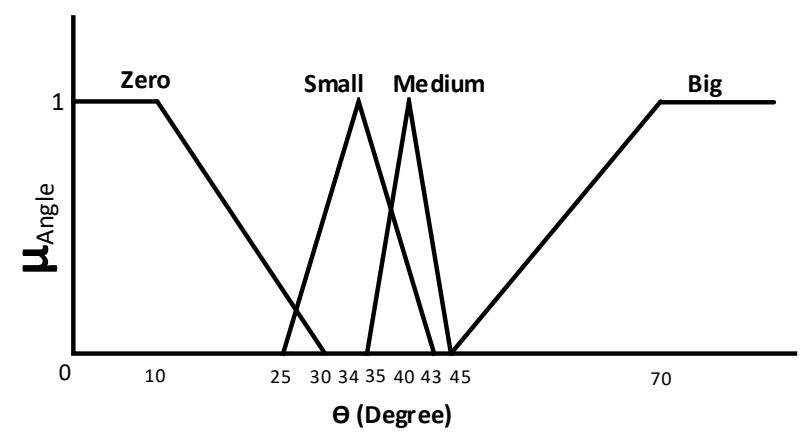

Figure 7. Angle Membership Function

a. Zero

$$
\mu_{\text {zero }}(\theta)=\left\{\begin{array}{cc}
1 ; & \theta \leq 10^{\circ} \\
\frac{30^{\circ}-\theta}{30-10} ; & 10 \leq \theta \leq 30 \\
0 ; & \theta \geq 30
\end{array}\right.
$$

b. Small

$$
\mu_{\text {small }}(\theta)=\left\{\begin{array}{lc}
0 ; \theta \leq 25 \text { and } \theta x \geq 43 \\
\frac{\theta-25}{34-25} ; & 25 \leq \theta \leq 34 \\
\frac{43-\theta}{43-34} ; & 34 \leq \theta \leq 43
\end{array}\right.
$$

c. Medium

$$
\mu_{\text {medium }}(\theta)=\left\{\begin{array}{c}
0 ; \quad \theta \leq 35 \text { and } \theta \geq 45 \\
\frac{\theta-35}{40-35} ; 35 \leq \theta \leq 40 \\
\frac{45-\theta}{45-40} ; 40 \leq \theta \leq 45
\end{array}\right.
$$

d. Big

$$
\mu_{b i g}(\theta)=\left\{\begin{array}{rc}
0 ; & \theta \leq 45 \\
\frac{\theta-45}{70-45} ; & 45 \leq \theta \leq 70 \\
1 ; & \theta \geq 70
\end{array}\right.
$$

\subsection{Fuzzy Rules}

Each parameter to define the plan, initialized to the input crips. This input cache receives input from the sensor used. The use of distance and angle sensor parameters initialized on the input crips aims to set the motor's rotational speed using a fuzzy logic control system. The use of distance and angle parameters based on the fact that in RCPS there is a change of rotation and rotation motion repeatedly. Rules in the system are 
created based on a combination of parameters already applied to the membership function.Variables set and fuzzy rules can be seen in Figure 8.

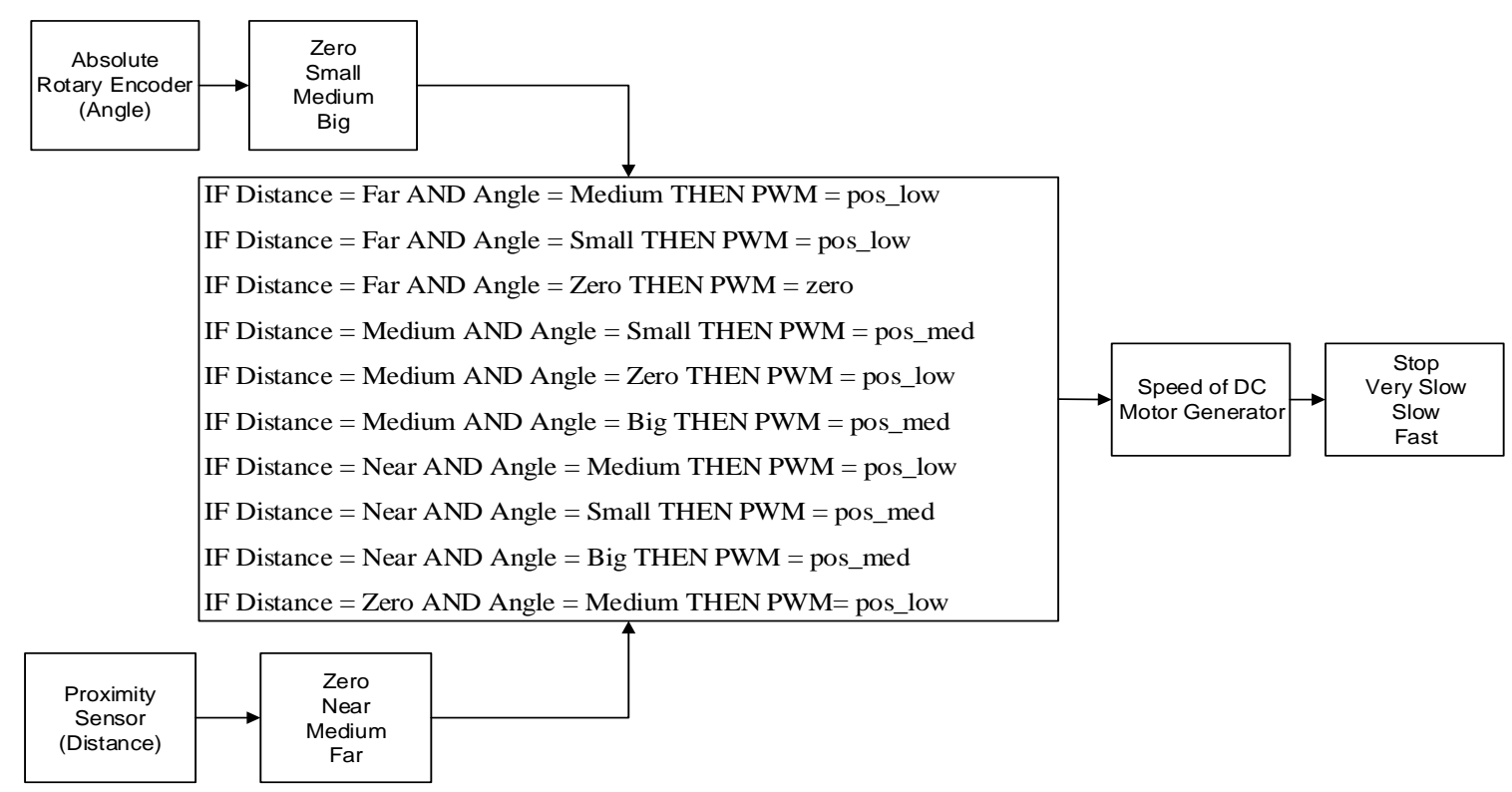

Figure 8. Variables, set and fuzzy rules

Rules are the speed controller by determining the value of PWM motor, where the provision of its PWM value as follows: pos $_{\text {high }}=255 \rightarrow$ fast, pos $_{\text {med }}=200 \rightarrow$ slow, pos low $_{1}=150 \rightarrow$ very slow, zero $=90 \rightarrow$ stop. With PWM equals to 90 , the motor is not moving because of the existing load. In performing fuzzy system inference, MAX method is used, where rules $[i][j]=$ $\max (\operatorname{distance}[i]$, angle $[j])$.

\subsection{Defuzzification}

The process maps the magnitudes of the fuzzy set into crisp values, since the regulated system is a real quantity, not fuzzy. The resulting value is then converted in PWM form and forwarded to the DC motor driver to adjust the rotational speed of the DC motor based on the given voltage. The defuzzification process uses the Center of Sum method.

\section{IMPLEMENTATION OF FUZZY LOGIC CONTROL SYSTEM}

The designed fuzzy logic control system is intended to control the speed of the Slot movement from one point to the next. As already explained, that the distance between the joint slots on the miniature RCPS of $14 \mathrm{~cm}$, then testing is done to the slot movement from one position to the next position. In this case the system is tested for the slot movement from the slot position C to the slot B position, since the ultrasonic sensor is placed in the positive $\mathrm{x}$-axis position (slot position B) (Figure 1). Rotation of RCPS on its realization clockwise. Before testing is done, all sensors are calibrated at first. Schematic process of RCPS control system with fuzzy logic can be seen in Figure 9, while the software flowchart is illustrated at Figure 10.

The ultrasonic sensor will work by emitting an ultrasonic signal to the object (Slot at position C) which is then reflected back to the sensor (refer to Figure 4). When the slot moves down, the system will record the change in distance to the position of the sensor. This distance data will be recorded on the system. The farthest distance is $14 \mathrm{~cm}$ and the nearest is $0 \mathrm{~cm}$. At the same time, the rotary encoder absolute will record the time and the abandoned angle $(\theta)$ due to the movement of this slot (refer to Figure 3 ). The recorded data is a decimal number ( 0 - 1023) which is then converted into angular form by Equation (12) as follows,

$$
\theta^{\circ}=\frac{\text { Decimal value } \times 360^{\circ}}{1024}
$$




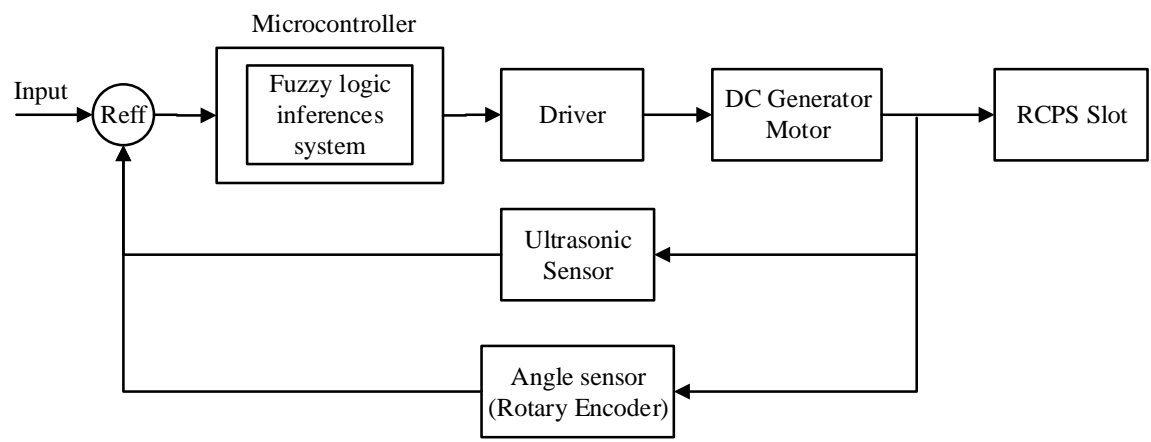

Figure 9. Proposed RCPS control system

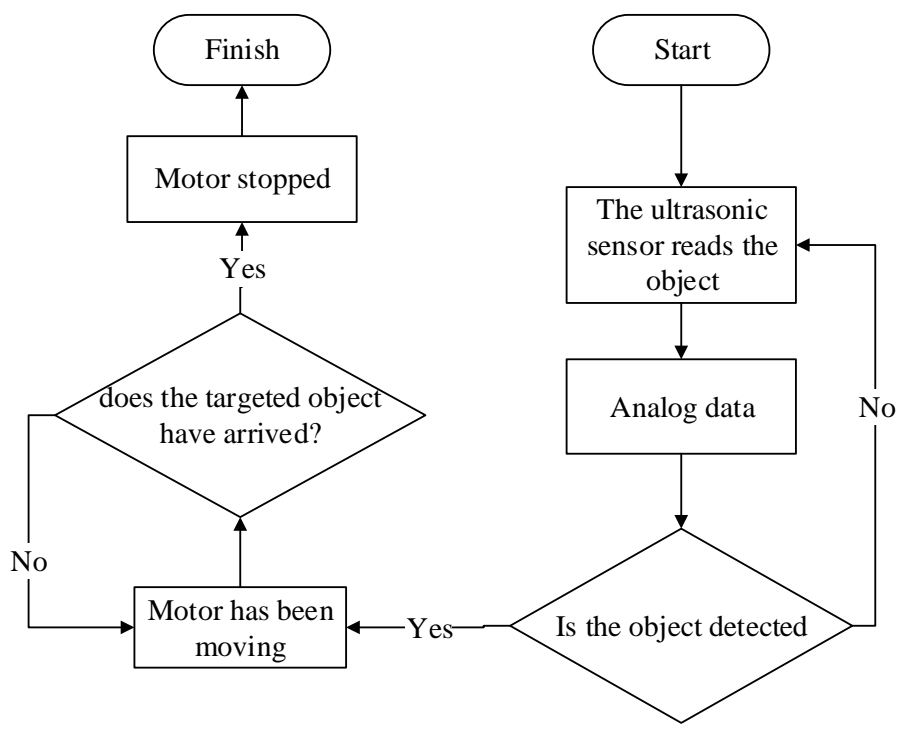

Figure 10. Testing Flowchart

\section{RESULTS AND DISCUSSION}

The proposed control system is evaluated either with or without fuzzy logic. The testing of a fuzzy no-control system begins with the reading of ultrasonic sensors to objects. The parameters set on the microcontroller based on the sensor are high (1) and low (0) because they do not use the reasoning approach stage. Input data on the sensor is in the form of analog data. The working principle of this system is when the sensor detects an object, the motor will spin to move the slot. If the object has arrived on the correct position, then the motor will stop. The motor response test using this absolute rotary encoder sensor has the reference value specified using Equation (1), in which $y_{1}=y_{\max } \cos \theta=42 \times \cos \theta$, while 42 is the max values of the $y$-axis based on Figure 3. Using the distance of slot C to slot B around $14 \mathrm{~cm}\left(y_{1}\right)$, then it can be deducted that $\theta_{\text {ref }}=\arccos \left(\frac{14}{42}\right)=70.5^{\circ} . \theta_{\text {ref }}$ is the maximum angle formed by the slot movement from point $\mathrm{C}$ to point $B$ (Figure 3). The test results of the system response can be seen in Figure 11. 


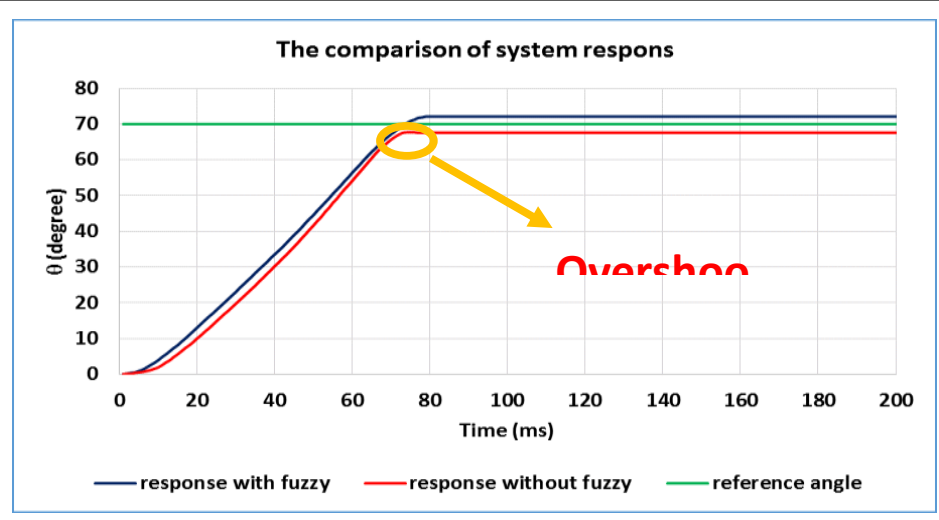

Figure 11. System Responses

The maximum measured maximum angle on testing the system without fuzzy control is $67.58^{\circ}$ with noticeable overshoot. Figure 11 also shows the system respon with fuzzy control, where the dc motor response uses a fuzzy control system that moves linearly until it reaches an angle of $72.14^{\circ}$. If both test results are compared, it can be concluded that the use of the fuzzy system on this RCPS control provides faster response and no overshoot, while the non fuzzy control system shows rather slower response and produces overshoot. Table 2 shows the performance comparison with and without fuzzy control system.

Table 2. Step Response

\begin{tabular}{lcc}
\hline Performance Parameter & Control system without Fuzzy & Control system with Fuzzy \\
\hline Rise Time & $0.58 \mathrm{~s}$ & $0.54 \mathrm{~s}$ \\
Peak Time & $0.85 \mathrm{~s}$ & - \\
Setling Time & $0.89 \mathrm{~s}$ & $0.83 \mathrm{~s}$ \\
Overshoot & $0.20 \%$ & - \\
Steady State Error & $4.14 \%$ & $2.32 \%$ \\
\hline
\end{tabular}

The fuzzy control system response step has a smaller rise time value compared to the fuzzy control system. This indicates that the initial response of the fuzzy control system is faster than the system response without fuzzy control. Control system without fuzzy there peak time value of 0.85 seconds due to the overshoot of $0.20 \%$ while the fuzzy control system there is no peak time value due to the absence of overshoot. The value of the reference angle that has been determined in the response step that is equal to 70.5. Based on the reference point value specified, there is an error value in the system response without fuzzy control of $4.14 \%$. While the fuzzy control system response there is an error value of $2.32 \%$. This proves that the fuzzy control system can be more optimally used in miniature rotary car parking systems than the non-fuzzy control system.

\section{CONCLUSION}

The results showed that the kinematics analysis before the implementation of the RCPS system helped provide more precise results. This is necessary because every form of RCPS will have an impact on the kinematics problem. The fuzzy control system with Sugeno inference model used in RCPS provides better results compared to conventional control. Step response on the fuzzy control system shows the rise time value of 0.58 seconds, peak time 0.85 seconds, 0.18 seconds settling time, $0.20 \%$ overshoot, and an angle error of $4.14 \%$. While the fuzzy control system the rise time value is 0.54 seconds, settling time 0.83 seconds, $2.32 \%$ error and the absence of peak time and overshoot. This makes the fuzzy control system can be more optimally used in miniature RCPS. Further research could be conducted including variation of simulation parameters, different implementation of RCPS, different control system, etc.

\section{REFERENCES}

[1] S. G. Narone, S. S. Chabukswar, S. A. Valyal, and R. B. Hirapure, "Vertical Car Parking - A Prototype," Int. J. Emerg. Technol. Adv. Eng, vol. 5, pp. 199-203, 2015. 
[2] P. Raman, A. Kumar, and R. P. Kushwaha, "Rotary Automated Car Parking System," Int. J. Sci. Eng. Technol. Res, vol. 5, pp. 1788-1791, 2016.

[3] L. A. Zadeh, "Fuzzy logic — a personal perspective.," in Fuzzy Sets and Systems: Science Direct, 2015, pp. 4-20.

[4] E. M. C. Mendoza, R. R. Perez, and J. J. S. Moriano, "Design of Neuro-Fuzzy Controller for Control of Water Distribution in an Irrigation Main Canal," IEEE Latin America Transactions, vol. 4, pp. 471-476, 2016.

[5] X.-G. Guo, J.-L. Wang, and F. Liao, "Adaptive fuzzy fault-tolerant control for multiple high-speed trains with proportional and integral-based sliding mode," IET Control Theory \& Applications (vol. 11, pp. 1234-1244, 2017.

[6] C. C. Lee, "Fuzzy Logic in Control System: Fuzzy Logic Controller - Part I," IEEE Transactions on System, Man, and Cybernetics vol. 20, pp. 404-418, 1990.

[7] J. Fernández, P. Cortés, J. Muñuzuri, and J. Guadix, "Dynamic Fuzzy Logic Elevator Group Control System With Relative Waiting Time Consideration," IEEE Transactions on Industrial Electronics, vol. 61, pp. 4912-4919, 2014.

[8] B. M. Patre, P. S. Londhe, and R. M. Nagarale, "Fuzzy Sliding Mode Control for Spatial Control of Large Nuclear Reactor," IEEE Transactions on Nuclear Science, vol. 62, pp. 2255-2265, 2015.

[9] M.-K. Tsay, Z.-S. Lee, and C.-H. Liao, "Fuzzy Power Control for Downlink CDMA-Based LMDS Network," IEEE Transactions on Vehicular Technology, vol. 57, pp. 3917-3921, 2008.

[10] Z. Guoqiang, L. Zhao, and L. Jishun, "Optimization of an Intelligent Controller for Parallel Autonomous Parking," TELKOMNIKA, vol. 11, pp. 1069-1075, 2013.

[11] T. Wang, L. Qu, H. Chen, and J. Wu, "Simulate Study of Automatic Parking System," TELKOMNIKA, vol. 11, pp. 7324-7330, 2013

[12] E. H. Mamdani, "Application of fuzzy algorithms for control of simple dynamic plant," in Proceedings IEEE, pp. $1585-1588,1974$.

[13] E. H. Mamdani and S. Assilian, "An experiment in linguistic synthesis with a fuzzy logic controller," International Journal of Man-Machine Studies, vol. 7, pp. 1-13, 1975.

[14] E. H. Mamdani and N. Baaklini, "Prescriptive method for deriving control policy in a fuzzy logic control," Electronic Lett. Ii, pp. 625-626, 1975.

[15] P. A. Adewuyi, "Performance Evaluation of Mamdani-type and Sugeno-type Fuzzy Inference System Based Controllers for Computer Fan," International Journal Information Technology and Computer Science, vol. 5, pp. 26-36, 2013.

[16] B. Jiang, Z. Gao, P. Shi, and Y. Xu, "Adaptive Fault-Tolerant Tracking Control of Near-Space Vehicle Using Takagi-Sugeno Fuzzy Models," IEEE TRANSACTIONS ON FUZZY SYSTEMS, vol. 12, pp. 1000-1007, 2010.

[17] T. Takagi and M. Sugeno, "Derivation of fuzzy control rules from human operators actions," in Proceedings of the IFAC Symposium on Fuzzy Information, Marseille, pp. 55-60, 1983.

[18] H. Mansor, A. N. Azlin, T. S. Gunawan, M. M. Kamal, and A. Z. Hashim, "Development of Smart Chicken Poultry Farm," Indonesian Journal of Electrical Engineering and Computer Science, vol. 10, pp. 498-505, 2018.

[19] J. S. Roger, C. T. Sun, and E. Mizutani, Neuro-fuzzy and soft computing: a computational approach to learning and machine intelligence. Upper Saddle River, Prentice Hall, 2008. 